\title{
Adaptive Sliding Mode Control Based on Uncertainty and Disturbance Estimator
}

\author{
Yue Zhu and Sihong Zhu \\ College of Engineering, Nanjing Agricultural University, Nanjing 210031, China \\ Correspondence should be addressed to Yue Zhu; zhuyue_jin@163.com
}

Received 27 March 2014; Revised 1 June 2014; Accepted 11 June 2014; Published 28 August 2014

Academic Editor: Dan Ye

Copyright (C) 2014 Y. Zhu and S. Zhu. This is an open access article distributed under the Creative Commons Attribution License, which permits unrestricted use, distribution, and reproduction in any medium, provided the original work is properly cited.

\begin{abstract}
This paper presents an original adaptive sliding mode control strategy for a class of nonlinear systems on the basis of uncertainty and disturbance estimator. The nonlinear systems can be with parametric uncertainties as well as unmatched uncertainties and external disturbances. The novel adaptive sliding mode control has several advantages over traditional sliding mode control method. Firstly, discontinuous sign function does not exist in the proposed adaptive sliding mode controller, and it is not replaced by saturation function or similar approximation functions as well. Therefore, chattering is avoided in essence, and the chattering avoidance is not at the cost of reducing the robustness of the closed-loop systems. Secondly, the uncertainties do not need to satisfy matching condition and the bounds of uncertainties are not required to be unknown. Thirdly, it is proved that the closed-loop systems have robustness to parameter uncertainties as well as unmatched model uncertainties and external disturbances. The robust stability is analyzed from a second-order linear time invariant system to a nonlinear system gradually. Simulation on a pendulum system with motor dynamics verifies the effectiveness of the proposed method.
\end{abstract}

\section{Introduction}

Sliding mode control (SMC) is one of distinguished control methods because of its strong external disturbance rejection and parameter variations insensibility performance when matching condition holds. Since 1950s, SMC has attracted many attentions both in theory study and application area; see [1-7].

As known to all, one of the main obstacles for application of SMC is chattering, and when discontinuous term exists in control signal, chattering cannot avoid essentially. Many researches have proposed lots of methods to reduce or eliminate chattering. Reference [2] presented a chatteringfree second-order sliding mode control method for a class of multi-input systems. While [3] analyzed the chattering phenomenon in systems with second-order sliding modes. Since the amplitude of chattering is proportional to the discontinuity magnitude in control signal, adaptivity principles are employed to reduce the effect of chattering. Based on the evaluations of the equivalent control by a low-pass filter, [7] introduced an adaptation methodology for searching the minimum possible value of control. Reference [8] discussed sliding order and proposed "super-twist" controller, [9] proposed an adaptive sliding mode control for discrete-time systems. Reference [10] developed and discussed different SMC algorithms with adaptive process to tune control gain. In [11], the control gain is of varying magnitude according to an adaptation process, but the adaptation process is terminated once sliding mode starts.

In addition to chattering, some other disadvantages of SMC include that the bounds of uncertainty and external disturbances are required to be known usually and SMC merely guarantees complete robustness to uncertainties and external disturbances which satisfy matching condition. Hence, some researchers endeavor to improve SMC from these aspects. Reference [12] proposed a dynamical approach of sliding variable formulation, which can deal with unmatched uncertainties for a class of single-input linear systems, to achieve the asymptotical stability. Based on the thought of combining adaptive control and SMC, [13] presented an adaptive robust control of multi-input multioutput nonlinear systems transformable to two semistrict feedback forms. Reference [14] applied multiple-surface and adaptive backstepping design technique to a class of single-input 
single-output nonlinear systems and achieved asymptotical stability for the application to a single-link flexible-joint robot plant. Reference [15] introduced predictive control strategy into the design of SMC, and strong robustness to matched/unmatched uncertainties was possessed for a class of discrete time nonlinear uncertain coupled systems, on the condition that the change rate of uncertainty is bounded. Reference [16] proposed a robust control algorithm with sliding mode for stabilization of a three-axis stabilized flexible spacecraft in the presence of parametric uncertainty, external disturbances, and control input nonlinearity/dead zone. Reference [17] discussed an adaptive sliding mode control for a piezo-actuated stage. Reference [18] considered the development of constructive sliding mode control strategies based on measured output information only for linear, time-delay systems with bounded disturbances that are not necessarily matched. Reference [19] combined immersion and invariance (I\&I) adaptive scheme with SMC, in order to control a class of nonlinear systems with parametric uncertainties and unmatched external disturbance, while retaining discontinuous term in controller and requiring the bound of uncertainties. In [20], a controller design method based on uncertainty and disturbance estimator (UDE) was proposed for linear time invariant (LTI) systems. In [21], the results of [20] were extended to SMC; however, [21] only considered a class of single input single output linear plants with matched uncertainties.

So far, there is little adaptive SMC methods that can complete the following goals at the same time: (i) to avoid chattering in essence, (ii) to have strong robustness to parameter uncertainties as well as unmatched model uncertainties and external disturbances, and (iii) to avoid knowing the bounds of uncertainties. Besides, on one hand, a common way to eliminate chattering is replacing sign function $\operatorname{sgn}(\cdot)$ in the sliding mode controller with saturation function sat $(\cdot)$ or similar approximation approaches. However, such a way is at the cost of reducing the robustness of closed-loop systems. On the other hand, adaptive control methods often consider parameter uncertainties while SMC methods usually only have strong robustness to matched uncertainties. Therefore, the study of parameter uncertainties, as well as unmatched model uncertainties and external disturbances at the same time, is not a trivial thing. When the bounds of uncertainties are unknown, the difficulty of the closed-loop system design is increased further.

The purpose of this paper is to eliminate chattering fundamentally and to deal with parameter uncertainties as well as unmatched model uncertainties and external disturbances without requiring to know the bound of uncertainties.

In order to realize the objective, a novel uncertainty and disturbance estimator based adaptive sliding mode control (UDE-based ASMC) method is presented to avoid chattering in essence. According to I\&I adaptive control strategy, parameter uncertainties can be handled well, and a controller component which acts as an equivalent control is obtained. While applying uncertainty and disturbance estimator (UDE), another controller component which is used to deal with model uncertainties and external disturbances, is constructed. This controller component is continuous, sign function $\operatorname{sgn}(\cdot)$ does not appear, and no saturation function sat $(\cdot)$ or similar approximation approaches are employed; therefore, the notorious chattering is avoided essentially and it is not at the cost of reducing the robustness of closed-loop systems. Then by adding these two controller components together, the expected UDE-based adaptive sliding mode controller is obtained. The robust stability is analyzed from a second-order linear time invariant system to a nonlinear system gradually on the basis of Lyapunov stability theory.

The remainder of this paper is organized as follows. In Section 2, for a second-order LTI system, the basic ideology of the UDE-based ASMC is deduced, including the design of sliding mode, the constructing of parameter estimation law, and the obtaining of adaptive sliding mode controller. Then for a class of multi-input multioutput nonlinear systems, the corresponding UDE-based ASMC algorithm and robustness analysis are given in Section 3. Simulation on a pendulum system with motor dynamics is illustrated in Section 4. Section 5 draws the conclusions of the paper.

\section{Adaptive Sliding Mode Control for Second-Order Linear Time Invariant System}

First of all, consider the following second-order linear time invariant (LTI) system:

$$
\begin{aligned}
& \dot{x}_{1}=\alpha_{11} x_{1}+\alpha_{12}\left(x_{2}+x_{1} \theta\right), \\
& \dot{x}_{2}=\alpha_{21} x_{1}+\alpha_{22} x_{2}+u+\Delta d,
\end{aligned}
$$

where $x_{1} \in \mathbb{R}$ and $x_{2} \in \mathbb{R}$ are states, $u \in \mathbb{R}$ is input, $\theta$ is unknown constant parameter, $\Delta d$ denotes the unknown model uncertainties and external disturbances, and $\alpha_{i j}(i, j=$ $1,2)$ are known constants with $\alpha_{12} \neq 0$. Assume the change rate of $\Delta d$ is bounded; namely, $\dot{\Delta} d<D$, where $D$ is a known constant.

2.1. Sliding Mode Design. Define a sliding surface,

$$
\sigma=x_{2}-x_{2_{d}}
$$

On one hand, one can see from (1a) that there exists

$$
x_{2}=\frac{1}{\alpha_{12}}\left(-\alpha_{11} x_{1}-\alpha_{12} x_{1} \theta+\dot{x}_{1}\right) .
$$

On the other hand, the control objective is to realize that the equilibrium $x_{1}=0, x_{2}=0$ is stable.

Hence, let $\widetilde{\theta}=\widehat{\theta}+\beta\left(x_{1}\right)$ be the estimate of unknown parameter $\theta$ and

$$
z=\left(\widehat{\theta}+\beta\left(x_{1}\right)\right)-\theta=\widetilde{\theta}-\theta,
$$

where $z \in \mathbb{R}$ is parameter estimation error, $\widehat{\theta}$ and $\beta\left(x_{1}\right)$ are auxiliary variable and auxiliary function, respectively, and $\beta\left(x_{1}\right): \mathbb{R} \rightarrow \mathbb{R}$ is a smooth function.

Thus, to construct $x_{2_{d}}$ in (2),

$$
x_{2_{d}}=\frac{1}{\alpha_{12}}\left(-\alpha_{11}-k\right) x_{1}-x_{1} \tilde{\theta},
$$

where $k$ is a designable parameter. 
2.2. Parameter Estimation Law Design. According to I\&I adaptive control approach, it is suitable to suppose a parameter estimation law as

$$
\dot{\hat{\theta}}=\omega(x, \widehat{\theta}),
$$

where $x$ is state vector. For systems (1a) and (1b), $x=\left[x_{1}\right.$, $\left.x_{2}\right]^{\mathrm{T}}$.

Since $\theta$ is constant parameter, the derivative of $z$ is

$$
\dot{z}=\dot{\hat{\theta}}+\frac{\partial \beta\left(x_{1}\right)}{\partial x_{1}} \dot{x}_{1} .
$$

Due to that fact that (4) can be rewritten into

$$
\theta=\tilde{\theta}-z
$$

substituting (1a), (6), and (8) into (7) yields

$$
\begin{aligned}
\dot{z}= & \omega(x, \widehat{\theta})+\frac{\partial \beta\left(x_{1}\right)}{\partial x_{1}}\left[\alpha_{11} x_{1}+\alpha_{12}\left(x_{2}+x_{1} \theta\right)\right] \\
= & \omega(x, \widehat{\theta}) \\
& +\frac{\partial \beta\left(x_{1}\right)}{\partial x_{1}}\left\{\alpha_{11} x_{1}+\alpha_{12}\left[x_{2}+x_{1}(\widetilde{\theta}-z)\right]\right\} \\
= & \omega(x, \widehat{\theta})+\frac{\partial \beta\left(x_{1}\right)}{\partial x_{1}}\left[\alpha_{11} x_{1}+\alpha_{12}\left(x_{2}+x_{1} \tilde{\theta}\right)\right] \\
& -\frac{\partial \beta\left(x_{1}\right)}{\partial x_{1}} \alpha_{12} x_{1} z .
\end{aligned}
$$

Now, one can choose parameter estimation law as

$$
\omega(x, \widehat{\theta})=-\frac{\partial \beta\left(x_{1}\right)}{\partial x_{1}}\left[\alpha_{11} x_{1}+\alpha_{12}\left(x_{2}+x_{1} \widetilde{\theta}\right)\right] \text {. }
$$

Thus,

$$
\dot{z}=-\frac{\partial \beta\left(x_{1}\right)}{\partial x_{1}} \alpha_{12} x_{1} z
$$

2.3. Adaptive Sliding Mode Control Law Design. Let the required control be expressed as

$$
u=u_{0}+u_{n}
$$

Select Lyapunov function as

$$
V=\frac{1}{2} x_{1}^{2}+\frac{1}{2} \sigma^{2}+\frac{1}{2} z^{2}
$$

and then

$$
\dot{V}=x_{1} \dot{x}_{1}+\sigma \dot{\sigma}+z \dot{z} .
$$

Because of (2), (4), and (5), (1a) can be rewritten into

$$
\begin{aligned}
\dot{x}_{1}= & -k x_{1}+\alpha_{12}\left[x_{2}-\frac{1}{\alpha_{12}}\left(-\alpha_{11}-k\right) x_{1}-x_{1} \tilde{\theta}\right] \\
& -\alpha_{12} x_{1}(\widetilde{\theta}-\theta) \\
= & -k x_{1}+\alpha_{12}\left(x_{2}-x_{2_{d}}\right)-\alpha_{12} x_{1}(\tilde{\theta}-\theta) \\
= & -k x_{1}+\alpha_{12} \sigma-\alpha_{12} x_{1} z .
\end{aligned}
$$

Differentiating (2) and using (1a), (1b), (6), and (8) give

$$
\begin{aligned}
\dot{\sigma}= & \dot{x}_{2}-\dot{x}_{2_{d}} \\
= & \alpha_{21} x_{1}+\alpha_{22} x_{2}+u+\Delta d-\frac{\partial x_{2_{d}} \dot{\hat{\theta}}}{\partial \widehat{\theta}}-\frac{\partial x_{2_{d}}}{\partial x_{1}} \dot{x}_{1} \\
= & \alpha_{21} x_{1}+\alpha_{22} x_{2}-\frac{\partial x_{2_{d}} \dot{\hat{\theta}}}{\partial \widehat{\theta}} \\
& -\frac{\partial x_{2_{d}}}{\partial x_{1}}\left\{\alpha_{11} x_{1}+\alpha_{12}\left[x_{2}+x_{1}(\widetilde{\theta}-z)\right]\right\}+u+\Delta d \\
= & \Phi\left(x_{1}, x_{2}, \hat{\theta}, \dot{\hat{\theta}}\right)+\frac{\partial x_{2_{d}}}{\partial x_{1}} \alpha_{12} x_{1} z+u+\Delta d,
\end{aligned}
$$

where

$$
\begin{aligned}
\Phi\left(x_{1}, x_{2}, \hat{\theta}, \dot{\hat{\theta}}\right)= & \alpha_{21} x_{1}+\alpha_{22} x_{2}-\frac{\partial x_{2_{d}}}{\partial \hat{\theta}} \\
& -\frac{\partial x_{2_{d}}}{\partial x_{1}}\left\{\alpha_{11} x_{1}+\alpha_{12}\left[x_{2}+x_{1} \tilde{\theta}\right]\right\} .
\end{aligned}
$$

Choose

$$
u_{0}=-\lambda\left(\frac{\partial x_{2_{d}}}{\partial x_{1}}\right)^{2} \sigma-\Phi\left(x_{1}, x_{2}, \hat{\theta}, \dot{\hat{\theta}}\right)-\alpha_{12} x_{1},
$$

where $\lambda>0$ is a designable parameter.

Then

$$
\begin{aligned}
\dot{\sigma}= & \Phi\left(x_{1}, x_{2}, \hat{\theta}, \dot{\hat{\theta}}\right)+\frac{\partial x_{2_{d}}}{\partial x_{1}} \alpha_{12} x_{1} z+\left(u_{0}+u_{n}\right)+\Delta d \\
= & \Phi\left(x_{1}, x_{2}, \hat{\theta}, \dot{\hat{\theta}}\right)+\frac{\partial x_{2_{d}}}{\partial x_{1}} \alpha_{12} x_{1} z-\lambda\left(\frac{\partial x_{2_{d}}}{\partial x_{1}}\right)^{2} \sigma \\
& -\Phi\left(x_{1}, x_{2}, \hat{\theta}, \dot{\hat{\theta}}\right)-\alpha_{12} x_{1}+u_{n}+\Delta d \\
= & \frac{\partial x_{2_{d}}}{\partial x_{1}} \alpha_{12} x_{1} z-\lambda\left(\frac{\partial x_{2_{d}}}{\partial x_{1}}\right)^{2} \sigma-\alpha_{12} x_{1}+u_{n} \\
& +\Delta d .
\end{aligned}
$$

Substituting (11), (15), and (19) into (14) yields

$$
\begin{aligned}
\dot{V}= & x_{1}\left(-k x_{1}+\alpha_{12} \sigma-\alpha_{12} x_{1} z\right) \\
& +\sigma\left[\frac{\partial x_{2_{d}}}{\partial x_{1}} \alpha_{12} x_{1} z-\lambda\left(\frac{\partial x_{2_{d}}}{\partial x_{1}}\right)^{2} \sigma-\alpha_{12} x_{1}+u_{n}+\Delta d\right] \\
& +z\left(-\frac{\partial \beta\left(x_{1}\right)}{\partial x_{1}} \alpha_{12} x_{1} z\right) \\
= & -k x_{1}^{2}-\alpha_{12} x_{1}^{2} z-\frac{\partial \beta\left(x_{1}\right)}{\partial x_{1}} \alpha_{12} x_{1} z^{2}+\frac{\partial x_{2_{d}}}{\partial x_{1}} \alpha_{12} x_{1} z \sigma \\
& -\lambda\left(\frac{\partial x_{2_{d}}}{\partial x_{1}}\right)^{2} \sigma^{2}+\sigma\left(u_{n}+\Delta d\right) .
\end{aligned}
$$


Here, we select

$$
\beta\left(x_{1}\right)=\frac{1}{2} \gamma \alpha_{12} x_{1}^{2}
$$

where $\gamma>0$ is a designable parameter.

Then

$$
\frac{\partial \beta\left(x_{1}\right)}{\partial x_{1}}=\gamma \alpha_{12} x_{1}
$$

With (22), (20) can be rewritten into

$$
\begin{aligned}
\dot{V}= & -k x_{1}^{2}-\gamma \alpha_{12}^{2} x_{1}^{2} z^{2}-\lambda\left(\frac{\partial x_{2_{d}}}{\partial x_{1}}\right)^{2} \sigma^{2}-\alpha_{12} x_{1}^{2} z \\
& +\frac{\partial x_{2_{d}}}{\partial x_{1}} \alpha_{12} x_{1} z \sigma+\sigma\left(u_{n}+\Delta d\right) .
\end{aligned}
$$

Because

$$
\begin{aligned}
x_{1}\left(\alpha_{12} z\right)\left(\sigma \frac{\partial x_{2_{d}}}{\partial x_{1}}\right) \leq & \frac{1}{2}\left[\left(x_{1} \alpha_{12} z\right)^{2}+\left(\sigma \frac{\partial x_{2_{d}}}{\partial x_{1}}\right)^{2}\right] \\
\leq & \frac{1}{2}\left[\left(x_{1} \alpha_{12} z\right)^{2}+\left(\sigma \frac{\partial x_{2_{d}}}{\partial x_{1}}\right)^{2}\right] \\
& +\frac{1}{2} x_{1}^{2}\left[1+\left(\alpha_{12} z\right)\right]^{2} \\
= & \frac{1}{2}\left[\left(x_{1} \alpha_{12} z\right)^{2}+\left(\sigma \frac{\partial x_{2_{d}}}{\partial x_{1}}\right)^{2}\right] \\
& +\frac{1}{2} x_{1}^{2}\left[1+2\left(\alpha_{12} z\right)+\left(\alpha_{12} z\right)^{2}\right] \\
= & \frac{1}{2}\left[\left(x_{1} \alpha_{12} z\right)^{2}+\left(\sigma \frac{\partial x_{2_{d}}}{\partial x_{1}}\right)^{2}\right] \\
& +\frac{1}{2} x_{1}^{2}\left[1+\left(\alpha_{12} z\right)^{2}\right]+\alpha_{12} x_{1}^{2} z
\end{aligned}
$$

namely,

$$
\begin{aligned}
- & \alpha_{12} x_{1}^{2} z+\frac{\partial x_{2_{d}}}{\partial x_{1}} \alpha_{12} x_{1} z \sigma \\
\leq & \frac{1}{2}\left[\left(x_{1} \alpha_{12} z\right)^{2}+\left(\sigma \frac{\partial x_{2_{d}}}{\partial x_{1}}\right)^{2}\right] \\
& +\frac{1}{2} x_{1}^{2}\left[1+\left(\alpha_{12} z\right)^{2}\right] \\
= & \frac{1}{2} x_{1}^{2}+\left(x_{1} \alpha_{12} z\right)^{2}+\frac{1}{2}\left(\sigma \frac{\partial x_{2_{d}}}{\partial x_{1}}\right)^{2},
\end{aligned}
$$

by using inequality (25), there exists

$$
\begin{aligned}
\dot{V} \leq & -k x_{1}^{2}-\gamma \alpha_{12}^{2} x_{1}^{2} z^{2}-\lambda\left(\frac{\partial x_{2_{d}}}{\partial x_{1}}\right)^{2} \sigma^{2}+\frac{1}{2} x_{1}^{2} \\
& +\left(x_{1} \alpha_{12} z\right)^{2}+\frac{1}{2}\left(\sigma \frac{\partial x_{2_{d}}}{\partial x_{1}}\right)^{2}+\sigma\left(u_{n}+\Delta d\right) \\
= & -\left(k-\frac{1}{2}\right) x_{1}^{2}-(\gamma-1) \alpha_{12}^{2} x_{1}^{2} z^{2} \\
& -\left(\lambda-\frac{1}{2}\right)\left(\frac{\partial x_{2_{d}}}{\partial x_{1}}\right)^{2} \sigma^{2}+\sigma\left(u_{n}+\Delta d\right) .
\end{aligned}
$$

According to Lyapunov stability theory, the derivative of the Lyapunov function should be negative in order to guarantee the stability of systems. Therefore, it is expected that the term $\sigma\left(u_{n}+\Delta d\right)$ in the right side of (26) is negative. One way to realize the expectation is to let the following be held:

$$
u_{n}+\Delta d=-\varsigma \sigma,
$$

where $\varsigma>0$ is a designable parameter.

Rewrite (27) into

$$
\Delta d=-\varsigma \sigma-u_{n} .
$$

Clearly, $\Delta d$ can be computed from the right hand side of (28); however, it cannot be directly used to obtain control component $u_{n}$. The uncertainty and disturbance estimator(UDE-) based control strategy proposed in [20] adopts an estimation of unknown model uncertainties and external disturbances $\Delta d$ to construct control laws. In the following, the UDE-based control strategy will be employed to obtain control component $u_{n}$.

Suppose $u d e$ is the estimate of $\Delta d$; by utilizing (28), ude can be accurately estimated as

$$
u d e=\left(-\varsigma \sigma-u_{n}\right) \star g_{f}(t),
$$

where " $\star$ " is the convolution operator, with

$$
G_{f}(s)=\mathscr{L}\left\{g_{f}(t)\right\},
$$

and $\mathscr{L}\{\cdot\}$ is the Laplace transform operator. Assume $g_{f}(t)$ is the impulse response of a strictly proper filter $G_{f}(s)$.

Now, the estimate variable $u d e$ enables the design of $u_{n}$ as

$$
u_{n}=-u d e,
$$

namely,

$$
u_{n}=\left(\varsigma \sigma+u_{n}\right) \star g_{f}(t) ;
$$

hence,

$$
u_{n}=\varsigma \sigma \star \frac{g_{f}(t)}{1-g_{f}(t)}=\varsigma \sigma \mathscr{L}^{-1}\left\{\frac{G_{f}(s)}{1-G_{f}(s)}\right\} .
$$

Because $u d e$ is the estimate of $\Delta d$, there exists

$$
u d e \approx \Delta d
$$


thus,

$$
u_{n} \approx-\Delta d
$$

therefore, (26) can be simplified to

$$
\begin{aligned}
\dot{V} \leq & -\left(k-\frac{1}{2}\right) x_{1}^{2}-(\gamma-1) \alpha_{12}^{2} x_{1}^{2} z^{2} \\
& -\left(\lambda-\frac{1}{2}\right)\left(\frac{\partial x_{2_{d}}}{\partial x_{1}}\right)^{2} \sigma^{2} .
\end{aligned}
$$

Hence, $\dot{V} \leq 0$ will be held, if design parameters are chosen as

$$
\begin{aligned}
& k>\frac{1}{2}, \\
& \gamma>1, \\
& \lambda>\frac{1}{2}, \\
& \varsigma>0 .
\end{aligned}
$$

Up to now, adaptive sliding mode control law for secondorder LTI system is obtained:

$$
\begin{aligned}
u= & -\lambda\left(\frac{\partial x_{2_{d}}}{\partial x_{1}}\right)^{2} \sigma-\Phi\left(x_{1}, x_{2}, \hat{\theta}, \dot{\hat{\theta}}\right)-\alpha_{12} x_{1} \\
& +\varsigma \sigma \mathscr{L}^{-1}\left\{\frac{G_{f}(s)}{1-G_{f}(s)}\right\} .
\end{aligned}
$$

Theorem 1. For second-order LTI systems (1a) and (1b), the equilibrium $\left[x_{1}^{*}, \sigma^{*}\right]^{\mathrm{T}}=[0,0]^{\mathrm{T}}$ is globally asymptotically stable under the adaptive sliding mode controller (38), with adaptive linear sliding modes (2), (5), and parameter estimation laws (6), (10), and (21), and design parameters $k, \gamma, \lambda, \varsigma$ satisfy (37).

Proof. According to the above derivation process, the results can be directly obtained from Barbashin-Krasovskii theorem [22].

Remark 2. The above result is based on the premise that (34) holds. Here, we analyse the accuracy of estimation briefly. Generally speaking, the low-pass filter $G_{f}(s)$ can be chosen by designers arbitrarily; however, it is practical to select $G_{f}(s)$ to be of a simple form such as

$$
G_{f}(s)=\frac{1}{T s+1},
$$

where $T=1 / \omega_{f}$ is a small positive constant. Thus,

$$
1-G_{f}(s)=\frac{T s}{T s+1}=T s G_{f}(s) .
$$

Define the error in estimation as

$$
e=\Delta d-u d e .
$$

With the above $G_{f}(s)$ and in view of (28), (29), and (41), it gives

$$
\begin{aligned}
e & =\left(-\varsigma \sigma-u_{n}\right) \star\left(1-g_{f}(t)\right)=\Delta d \star\left(1-g_{f}(t)\right) \\
& =\Delta d \mathscr{L}^{-1}\left\{T s G_{f}(s)\right\}=T \dot{\Delta} d \mathscr{L}^{-1}\left\{G_{f}(s)\right\} .
\end{aligned}
$$

Therefore, (34) will hold, if the term $T \dot{\Delta} d$ is sufficiently small. Remark 3. When the low-pass filter (39) is employed, because

$$
\frac{G_{f}(s)}{1-G_{f}(s)}=\frac{1}{T s}
$$

and (33), the control component $u_{n}$ can be simplified to

$$
u_{n}=\varsigma \sigma \mathscr{L}^{-1}\left\{\frac{1}{T s}\right\}=\frac{\varsigma}{T} \int_{0}^{t} \sigma d \tau .
$$

From (44), it is easy to find that an integral action is included in the controller (38). In traditional SMC method, $\operatorname{sgn}(\sigma)$ is used to deal with uncertainties and disturbances. However, there is no such a discontinuous term in our method, an integral term instead. Therefore, chattering is eliminated essentially.

Remark 4. The smooth function $\beta\left(x_{1}\right)$ in (21) is only one of choices for the system. According to the Lyapunov stability theory, any smooth function $\beta\left(x_{1}\right)$ which makes the derivative of the Lyapunov function negative is applicable.

\section{Adaptive Sliding Mode Control for Nonlinear System}

Consider the following nonlinear system, which is with parametric uncertainties as well as unmatched model uncertainties and external disturbances,

$$
\begin{aligned}
& \dot{\mathbf{x}}_{1}=\mathbf{f}_{0}\left(\mathbf{x}_{1}\right)+\mathbf{f}_{1}\left(\mathbf{x}_{1}\right) \boldsymbol{\theta}+\mathbf{g}_{1}\left(\mathbf{x}_{1}\right) \mathbf{x}_{2}, \\
& \dot{\mathbf{x}}_{2}=\mathbf{f}_{2}(\mathbf{x})+\mathbf{g}_{2}(x) \mathbf{u}+\Delta \mathbf{F}(\mathbf{x}, t)
\end{aligned}
$$

where $\mathbf{x}=\left[\mathbf{x}_{1}, \mathbf{x}_{2}\right]^{\mathrm{T}}$ is state vector, $\mathbf{x}_{1} \in \mathbb{R}^{n-m}$, and $\mathbf{x}_{2} \in \mathbb{R}^{m}, \mathbf{u} \in \mathbb{R}^{m}$ is input vector, $\boldsymbol{\theta} \in \mathbb{R}^{q}$ is unknown constant parameter vector, $\Delta \mathbf{F}(\mathbf{x}, t)$ denotes the unknown unmatched model uncertainties and external disturbances, $\mathbf{f}_{0}\left(\mathbf{x}_{1}\right): \mathbb{R}^{n-m} \rightarrow \mathbb{R}^{n-m}$ and $\mathbf{f}_{2}(\mathbf{x}): \mathbb{R}^{n} \rightarrow \mathbb{R}^{m}$ are known nonlinear smooth function vectors, $\mathbf{f}_{1}\left(\mathbf{x}_{1}\right): \mathbb{R}^{n-m} \rightarrow$ $\mathbb{R}^{(n-m) \times q}$ and $\mathbf{g}_{1}\left(\mathbf{x}_{1}\right): \mathbb{R}^{n-m} \rightarrow \mathbb{R}^{(n-m) \times m}$ are known nonlinear smooth function matrices, and $\mathbf{g}_{2}(\mathbf{x}): \mathbb{R}^{n} \rightarrow$ $\mathbb{R}^{m \times m}$ is a known nonzero matrix.

In the following, when there is no confusion, we will use $\mathbf{f}_{1}$ instead of $\mathbf{f}_{1}\left(\mathbf{x}_{1}\right)$ for simplicity, and other functions are the same.

Theorem 5. For nonlinear systems (45a) and (45b), the closedloop system is globally asymptotically stable under the adaptive sliding mode controllers (46a), (46b), and (46c) with adaptive 
linear sliding modes (49a) and (49b) and parameter estimation law (50), if Assumptions 1, 2, 3, and 4 are held.

(1) Adaptive Sliding Mode Controller. Consider

$$
\begin{gathered}
\mathbf{u}=\mathbf{u}_{0}+\mathbf{u}_{n}, \\
\mathbf{u}_{0}=-\mathbf{g}_{2}^{+}\left(\lambda\left\|\frac{\partial \mathbf{x}_{2_{d}}}{\partial \mathbf{x}_{1}}\right\|^{2} \boldsymbol{\sigma}+\mathbf{g}_{1}^{\mathrm{T}} \frac{\partial V_{0}}{\partial \mathbf{x}_{1}}\right)-\Phi, \\
\mathbf{u}_{n}=\mathbf{g}_{2}^{+}\left[\left(1-g_{f}(t)\right)^{-1} g_{f}(t)\right] \star \boldsymbol{\varsigma} \boldsymbol{\sigma} \\
=\mathbf{g}_{2}^{+} \mathscr{L}^{-1}\left[\left(1-G_{f}(s)\right)^{-1} G_{f}(s)\right] \boldsymbol{\varsigma} \boldsymbol{\sigma},
\end{gathered}
$$

where $\lambda$ is designable parameters, $\Phi=\mathbf{g}_{2}^{+}\left[\mathbf{f}_{2}-\left(\partial \mathbf{x}_{2_{d}} / \partial \mathbf{x}_{1}\right)\left(\mathbf{f}_{0}+\right.\right.$ $\left.\left.\mathbf{f}_{1} \widetilde{\boldsymbol{\theta}}+\mathbf{g}_{1} \mathbf{x}_{2}\right)-\left(\partial \mathbf{x}_{2_{d}} / \partial \widehat{\boldsymbol{\theta}}\right) \dot{\hat{\boldsymbol{\theta}}}\right], \mathbf{g}_{2}^{+}=\left[\mathbf{g}_{2}^{\mathrm{T}} \mathbf{g}_{2}\right]^{-1} \mathbf{g}_{2}^{\mathrm{T}}$ is the pseudoinverse of $\mathbf{g}_{2}, \widetilde{\boldsymbol{\theta}}=\widehat{\boldsymbol{\theta}}+\boldsymbol{\beta}$ is the estimate of $\boldsymbol{\theta}, \widehat{\boldsymbol{\theta}}$ is auxiliary variable, $\boldsymbol{\beta}(\cdot): \mathbb{R}^{n-m} \rightarrow \mathbb{R}^{q}$ is a smooth function with respect to $\mathbf{x}_{1}$, and $\mathbf{z}$ is parameter estimation error defined as

$$
\mathbf{z}=\tilde{\boldsymbol{\theta}}-\boldsymbol{\theta}
$$

$\boldsymbol{\sigma} \in \mathbb{R}^{m}$ is sliding mode variable, and $\boldsymbol{\varsigma} \boldsymbol{\sigma}$ is defined as

$$
\boldsymbol{\varsigma} \boldsymbol{\sigma}=\left[\begin{array}{c}
\varsigma_{1} \sigma_{1} \\
\varsigma_{2} \sigma_{2} \\
\vdots \\
\varsigma_{m} \sigma_{m}
\end{array}\right] .
$$

$\varsigma_{i}>0(i=1,2, \ldots, m)$ are designable parameters.

(2) Adaptive Sliding Mode. Consider

$$
\begin{aligned}
\boldsymbol{\sigma} & =\mathbf{x}_{2}-\mathbf{x}_{2_{d}}, \\
\mathbf{x}_{2_{d}} & =\boldsymbol{v}\left(\mathbf{x}_{1}, \widetilde{\boldsymbol{\theta}}\right) .
\end{aligned}
$$

(3) Parameter Estimation Law. Consider

$$
\dot{\hat{\boldsymbol{\theta}}}=-\frac{\partial \boldsymbol{\beta}}{\partial \mathbf{x}_{1}}\left(\mathbf{f}_{0}+\mathbf{f}_{1} \tilde{\boldsymbol{\theta}}+\mathbf{g}_{1} \mathbf{x}_{2}\right) .
$$

\section{(4) Assumptions}

Assumption 1. There exists a full information bounded control law $\boldsymbol{\nu}\left(\mathbf{x}_{1}, \boldsymbol{\theta}\right)$ which satisfies Lipschitz condition:

$$
\left\|\nu\left(\mathbf{x}_{1}, \boldsymbol{\theta}+\zeta\right)-\nu\left(\mathbf{x}_{1}, \boldsymbol{\theta}\right)\right\| \leq \mathrm{M}\|\zeta\|
$$

for all $\zeta \in \mathbb{R}^{q}$ and for certain function $\mathbf{M}\left(\mathbf{x}_{1}\right)$, where $\mathbf{M}(\cdot)$ : $\mathbb{R}^{n} \rightarrow \mathbb{R}>0$, such that the closed-loop system

$$
\dot{\mathbf{x}}_{1}=\mathbf{f}_{0}+\mathbf{f}_{1} \boldsymbol{\theta}+\mathbf{g}_{1} \boldsymbol{v}\left(\mathbf{x}_{1}, \boldsymbol{\theta}\right) \triangleq \mathbf{f}^{*}\left(\mathbf{x}_{1}\right)
$$

has a globally asymptotically stable equilibrium at $\mathbf{x}_{1}^{*}$ with a radially unbounded function $V_{0}\left(\mathbf{x}_{1}\right)$ satisfying

$$
\frac{\partial V_{0}}{\partial \mathbf{x}_{1}} \mathbf{f}^{*}+L\left\|\frac{\partial V_{0}}{\partial \mathbf{x}_{1}} \mathbf{g}_{1}\right\|^{2} \leq 0
$$

for certain $0<L<\infty$.
Assumption 2. $\boldsymbol{\beta}(\cdot)$ satisfies

$$
\frac{\partial \boldsymbol{\beta}}{\partial \mathbf{x}_{1}} \mathbf{f}_{1}+\left(\frac{\partial \boldsymbol{\beta}}{\partial \mathbf{x}_{1}} \mathbf{f}_{1}\right)^{\mathrm{T}} \geq \mathbf{M}^{2} \mathbf{I}_{q}+\mathbf{f}_{1}^{\mathrm{T}} \mathbf{f}_{1} .
$$

Assumption 3. Designable parameters $\lambda, \delta, \mu, \epsilon$, and $\varsigma$ are chosen to satisfy

$$
\begin{aligned}
& \mu>0, \\
& \epsilon>0, \\
& \varsigma_{i}>0, \quad(i=1,2, \ldots, m), \\
& \lambda>\frac{1}{2 \epsilon}, \\
& \delta>\mu+\epsilon .
\end{aligned}
$$

Assumption 4. The change rate of $\Delta \mathbf{F}_{i}(\mathbf{x}, t),(i=1,2, \ldots, m)$ is bounded; namely, $\dot{\Delta} \mathbf{F}_{i}(\mathbf{x}, t)<\mathscr{F}_{i}, \mathscr{F}_{i},(i=1,2, \ldots, m)$ are known constants.

Proof. Based on the UDE method, the way to obtain control component $\mathbf{u}_{n}$ for nonlinear systems (45a) and (45b) is similar to that of Section 2.

Equation (56) is expected to be held:

$$
\mathbf{g}_{2} \mathbf{u}_{n}+\Delta \mathbf{F}=-\boldsymbol{\varsigma} \boldsymbol{\sigma} .
$$

Rewrite (56) into

$$
\Delta \mathbf{F}=-\boldsymbol{\varsigma} \boldsymbol{\sigma}-\mathbf{g}_{2} \mathbf{u}_{n} .
$$

Suppose ude is the estimate of $\Delta \mathbf{F}$; by utilizing (57), ude can be accurately estimated as

$$
\mathbf{u d e}=\left(-\boldsymbol{\varsigma} \boldsymbol{\sigma}-\mathbf{g}_{2} \mathbf{u}_{n}\right) \star g_{f}(t) .
$$

Now, the estimate variable ude enables the design of $\mathbf{u}_{n}$ as

$$
\mathbf{g}_{2} \mathbf{u}_{n}=-\mathbf{u d e}
$$

hence, it is easy to get $\mathbf{u}_{n}$ as shown in (46c).

Because $\mathbf{u d e}$ is the estimate of $\Delta \mathbf{F}$, there exists

$$
\text { ude } \approx \Delta \mathrm{F}
$$

thus, according to (59), it is easy to get

$$
\mathbf{g}_{2} \mathbf{u}_{n} \approx-\Delta \mathbf{F} \text {. }
$$

Substituting (47), (49a), (49b), and (52) into (45a) yields

$$
\begin{aligned}
\dot{\mathbf{x}}_{1} & =\mathbf{f}_{0}+\mathbf{f}_{1} \boldsymbol{\theta}+\mathbf{g}_{1}\left(\boldsymbol{\sigma}+\mathbf{x}_{2_{d}}\right)=\mathbf{f}_{0}+\mathbf{f}_{1} \boldsymbol{\theta}+\mathbf{g}_{1}\left[\boldsymbol{\sigma}+\boldsymbol{v}\left(\mathbf{x}_{1}, \widetilde{\boldsymbol{\theta}}\right)\right] \\
& =\mathbf{f}_{0}+\mathbf{f}_{1} \boldsymbol{\theta}+\mathbf{g}_{1} \boldsymbol{v}\left(\mathbf{x}_{1}, \boldsymbol{\theta}\right)+\mathbf{g}_{1}\left[\boldsymbol{\sigma}+\boldsymbol{v}\left(\mathbf{x}_{1}, \widetilde{\boldsymbol{\theta}}\right)\right]-\mathbf{g}_{1} \boldsymbol{\nu}\left(\mathbf{x}_{1}, \boldsymbol{\theta}\right) \\
& =\mathbf{f}^{*}\left(\mathbf{x}_{1}\right)+\mathbf{g}_{1} \boldsymbol{\sigma}+\mathbf{g}_{1}\left[\boldsymbol{v}\left(\mathbf{x}_{1}, \widetilde{\boldsymbol{\theta}}\right)-\boldsymbol{v}\left(\mathbf{x}_{1}, \boldsymbol{\theta}\right)\right] \\
& =\mathbf{f}^{*}\left(\mathbf{x}_{1}\right)+\mathbf{g}_{1} \boldsymbol{\sigma}+\mathbf{g}_{1}\left[\boldsymbol{v}\left(\mathbf{x}_{1}, \boldsymbol{\theta}+\mathbf{z}\right)-\boldsymbol{v}\left(\mathbf{x}_{1}, \boldsymbol{\theta}\right)\right] .
\end{aligned}
$$


According to estimation error (47), parameter estimation law (50), and system dynamic (45a), the dynamic of estimation error $\mathbf{z}$ can be given by

$$
\begin{aligned}
\dot{\mathbf{z}}= & \dot{\hat{\boldsymbol{\theta}}}+\frac{\partial \boldsymbol{\beta}}{\partial \mathbf{x}_{1}} \dot{\mathbf{x}}_{1} \\
= & -\frac{\partial \boldsymbol{\beta}}{\partial \mathbf{x}_{1}}\left(\mathbf{f}_{0}+\mathbf{f}_{1} \widetilde{\boldsymbol{\theta}}+\mathbf{g}_{1} \mathbf{x}_{2}\right) \\
& +\frac{\partial \boldsymbol{\beta}}{\partial \mathbf{x}_{1}}\left[\mathbf{f}_{0}+\mathbf{f}_{1}(\widetilde{\boldsymbol{\theta}}-\mathbf{z})+\mathbf{g}_{1} \mathbf{x}_{2}\right] \\
= & -\frac{\partial \boldsymbol{\beta}}{\partial \mathbf{x}_{1}} \mathbf{f}_{1} \mathbf{z} .
\end{aligned}
$$

Based on (47), (49a), (45a), and (45b) the dynamic of sliding mode $\sigma$ can be rewritten into

$$
\begin{aligned}
\dot{\boldsymbol{\sigma}}= & \dot{\mathbf{x}}_{2}-\dot{\mathbf{x}}_{2_{d}}=\mathbf{f}_{2}+\mathbf{g}_{2} \mathbf{u}+\Delta \mathbf{F}-\frac{\partial \mathbf{x}_{2_{d}}}{\partial \widehat{\boldsymbol{\theta}}}-\frac{\partial \mathbf{x}_{2_{d}}}{\partial \mathbf{x}_{1}} \dot{\mathbf{x}}_{1} \\
= & \mathbf{f}_{2}+\mathbf{g}_{2} \mathbf{u}+\Delta \mathbf{F}-\frac{\partial \mathbf{x}_{2_{d}} \dot{\hat{\boldsymbol{\theta}}}}{\partial \widehat{\boldsymbol{\theta}}} \\
& -\frac{\partial \mathbf{x}_{2_{d}}}{\partial \mathbf{x}_{1}}\left[\mathbf{f}_{0}+\mathbf{f}_{1}(\widetilde{\boldsymbol{\theta}}-\mathbf{z})+\mathbf{g}_{1} \mathbf{x}_{2}\right] .
\end{aligned}
$$

Select Lyapunov function as

$$
V(\mathbf{x}, \boldsymbol{\sigma}, \mathbf{z})=V_{0}\left(\mathbf{x}_{1}\right)+\frac{1}{2} \boldsymbol{\sigma}^{\mathrm{T}} \boldsymbol{\sigma}+\frac{\delta}{2} \mathbf{z}^{\mathrm{T}} \mathbf{z},
$$

where $\delta>0$.

Then, due to (46a), (46b), (46c), (61), (62), (63), and (64) and Assumptions 1, 2, 3, and 4, one can get

$$
\begin{aligned}
\dot{V}= & \frac{\partial V_{0}}{\partial \mathbf{x}_{1}}\left\{\mathbf{f}^{*}+\mathbf{g}_{1}\left[\boldsymbol{\nu}\left(\mathbf{x}_{1}, \boldsymbol{\theta}+\mathbf{z}\right)-\boldsymbol{v}\left(\mathbf{x}_{1}, \boldsymbol{\theta}\right)\right]\right\}-\delta \mathbf{z}^{\mathrm{T}}\left(\frac{\partial \boldsymbol{\beta}}{\partial \mathbf{x}_{1}} \mathbf{f}_{1} \mathbf{z}\right) \\
& -\lambda\left\|\frac{\partial \mathbf{x}_{2_{d}}}{\partial \mathbf{x}_{1}}\right\|^{2} \boldsymbol{\sigma}^{\mathrm{T}} \boldsymbol{\sigma}+\boldsymbol{\sigma}^{\mathrm{T}}\left(\mathbf{g}_{2} \mathbf{u}_{n}+\Delta \mathbf{F}\right)+\boldsymbol{\sigma}^{\mathrm{T}} \frac{\partial \mathbf{x}_{2_{d}}}{\partial \mathbf{x}_{1}} \mathbf{f}_{1} \mathbf{z} \\
\leq & \frac{\partial V_{0}}{\partial \mathbf{x}_{1}} \mathbf{f}^{*}+\frac{1}{2 \mu}\left\|\frac{\partial V_{0}}{\partial \mathbf{x}_{1}} \mathbf{g}_{1}\right\|^{2}+\frac{\mu}{2} \mathbf{M}^{2}\|\mathbf{z}\|^{2}-\delta \mathbf{z}^{\mathrm{T}} \frac{\partial \boldsymbol{\beta}}{\partial \mathbf{x}_{1}} \mathbf{f}_{1} \mathbf{z} \\
& +\frac{\epsilon}{2} \mathbf{z}^{\mathrm{T}} \mathbf{f}_{1}^{\mathrm{T}} \mathbf{f}_{1} \mathbf{z}-\left(\lambda-\frac{1}{2 \epsilon}\right)\left\|\frac{\partial \mathbf{x}_{2_{d}}}{\partial \mathbf{x}_{1}}\right\|^{2} \boldsymbol{\sigma}^{\mathrm{T}} \boldsymbol{\sigma}<0 .
\end{aligned}
$$

Therefore, the closed-loop system is globally asymptotically stable under the presented UDE-based adaptive sliding mode control.

Remark 6. Assumptions 1 and 2 are the prior knowledge of the nonlinear system. They are reasonable assumptions, and readers can find them in [23].

Remark 7. In LTI systems (1a) and (1b), the equilibrium point is $\left[x_{1}^{*}, \sigma^{*}\right]^{\mathrm{T}}=[0,0]^{\mathrm{T}}$. According to (2) and (5), it is convenient to obtain $x_{2}^{*}=0$. However, in nonlinear systems (45a) and (45b), the equilibrium point is $\left[\mathbf{x}_{1}^{*}, \boldsymbol{\sigma}^{*}\right]^{\mathrm{T}}=[\mathbf{0}, \mathbf{0}]^{\mathrm{T}}$. Because of (49a) and (49b), $\mathbf{x}_{2}^{*}=\mathbf{x}_{2_{d}}^{*}=\boldsymbol{v}\left(\mathbf{x}_{1}^{*}, \widetilde{\boldsymbol{\theta}}^{*}\right)$. When $\mathbf{x}_{2_{d}}$ is bounded, the nonlinear systems (45a) and (45b) can converge to $\mathbf{x}_{1}^{*}=\mathbf{0}$ and realize that $\mathbf{x}_{2}^{*}$ is bounded.

Remark 8. Similar to that of Remarks 2 and 3, for nonlinear systems (45a) and (45b), with low-pass filter (39), (60) will hold if the term $T \dot{\Delta} F_{i}(i=1,2, \ldots, m)$ is sufficiently small and an integral action is included in

$$
\mathbf{u}_{n}=\mathbf{g}_{2}+\frac{1}{T} \int \boldsymbol{\varsigma} \boldsymbol{\sigma} d \tau
$$

Because discontinuous term $\operatorname{sgn}(\sigma)$, which often appears in conventional sliding mode control method, is displaced by integral term in our method, chattering is eliminated essentially.

From (67) and (56), (58), it is clear that smaller $T$ implies a smaller estimation error but a larger magnitude of control if $\boldsymbol{\sigma}$ is not small. Fortunately, $\boldsymbol{\varsigma}$ gives designer an option to obtain a satisfying compromise in this aspect.

\section{Simulation}

In order to verify the validity of the proposed UDE-based ASMC method, a pendulum with motor dynamics given in [12] is considered in the following:

$$
\begin{aligned}
& \dot{x}_{1}=x_{2}, \\
& \dot{x}_{2}=-a \sin \left(x_{1}\right)-q \cos \left(x_{1}\right)+x_{3}, \\
& \dot{x}_{3}=-x_{3}+u+d(x, t),
\end{aligned}
$$

where $a$ and $q$ represent constant unknown parameters to the pendulum and $d(x, t)$ denotes uncertainties and disturbances.

According to the form of (45a) and (45b), there exists

$$
\begin{aligned}
& \mathbf{x}_{1}=\left[\begin{array}{l}
x_{1} \\
x_{2}
\end{array}\right] \text {, } \\
& \mathbf{x}_{2}=x_{3} \text {, } \\
& \boldsymbol{\theta}=\left[\begin{array}{l}
a \\
q
\end{array}\right] \\
& \mathbf{f}_{0}=\left[\begin{array}{c}
x_{2} \\
0
\end{array}\right] \text {, } \\
& \mathbf{f}_{1}=\left[\begin{array}{cc}
0 & 0 \\
-\sin \left(x_{1}\right) & -\cos \left(x_{1}\right)
\end{array}\right]=\left[\begin{array}{l}
\mathbf{f}_{11} \\
\mathbf{f}_{12}
\end{array}\right] \text {, } \\
& \mathbf{g}_{1}=\left[\begin{array}{l}
0 \\
1
\end{array}\right] \text {, } \\
& \mathbf{f}_{2}=-x_{3} \text {, } \\
& \mathbf{g}_{2}=1 \text {, } \\
& \Delta \mathbf{F}=d
\end{aligned}
$$


Select

$$
\begin{aligned}
\mathbf{x}_{2_{d}} & =[-1,-k] \mathbf{x}_{1}-\mathbf{f}_{12} \tilde{\boldsymbol{\theta}}, \\
\frac{\partial \boldsymbol{\beta}}{\partial \mathbf{x}_{1}} & =\delta \mathbf{f}_{1}^{\mathrm{T}}, \\
V_{0} & =\frac{1}{2} \mathbf{x}_{1}^{\mathrm{T}} \mathbf{x}_{1},
\end{aligned}
$$

where $k \in \mathbb{R}>0$ and $\delta \in \mathbb{R}>0$ are designable parameters.

Then $\partial \mathbf{x}_{2_{d}} / \partial x_{1}=-1+\widetilde{\theta}_{1} \cos \left(x_{1}\right), \partial \mathbf{x}_{2_{d}} / \partial x_{2}=-k, \partial \mathbf{x}_{2_{d}} /$ $\partial \hat{\boldsymbol{\theta}}=\left[\sin \left(x_{1}\right), \cos \left(x_{1}\right)\right], \partial V_{0} / \partial \mathbf{x}_{1}=\mathbf{x}_{1}$, and $\boldsymbol{\beta}=\int(\partial \boldsymbol{\beta} /$ $\left.\partial \mathbf{x}_{1}\right) d \mathbf{x}_{1}=\delta \mathbf{f}_{1}^{\mathrm{T}} \mathbf{x}_{1}+\mathbf{C}\left(\mathbf{x}_{1}\right)$, where $\mathbf{C}\left(\mathbf{x}_{1}\right)$ is certain smooth function vector.

According to (39), (46a), (46b), (46c), (49a), and (49b), the parameter estimation law and adaptive sliding mode control law for system (68) are

$$
\begin{aligned}
\dot{\hat{\boldsymbol{\theta}}} & =-\delta \mathbf{f}_{1}^{\mathrm{T}}\left(\mathbf{f}_{0}+\mathbf{f}_{1} \widetilde{\boldsymbol{\theta}}+\mathbf{g}_{1} \mathbf{x}_{2}\right), \\
\mathbf{u}= & -\left(\lambda\left\|\frac{\partial \mathbf{x}_{2_{d}}}{\partial x_{1}}\right\|^{2} \boldsymbol{\sigma}+\mathbf{g}_{1}^{\mathrm{T}} \mathbf{x}_{1}\right)-\mathbf{f}_{2} \\
& +\frac{\partial \mathbf{x}_{2_{d}}}{\partial x_{1}}\left(\mathbf{f}_{0}+\mathbf{f}_{1} \widetilde{\boldsymbol{\theta}}+\mathbf{g}_{1} \mathbf{x}_{2}\right)+\mathbf{x}_{1}^{\mathrm{T}} \dot{\boldsymbol{\theta}} \\
& +\frac{1}{T} \int \boldsymbol{\varsigma} \boldsymbol{\sigma} d \tau .
\end{aligned}
$$

In Figures 1 and 2, solid line shows the presented method in this paper, while dot line shows the method given in [12].

Figures 1 and 2 show the result by employing the presented UDE-based ASMC method under $k=2, \delta=2$, $\lambda=0.1, \mathbf{C}=[0,0]^{\mathrm{T}}, a=11, q=4, d=5 x_{1} \sin (0.2 t)$, $T=0.01$, and $\varsigma=0.0003$.

To make comparison, simulation results with the method proposed by [12] are given in Figures 1 and 2 simultaneously, by selecting $p_{1}=2, p_{2}=1, p_{3}=2, \tau_{1}=2, \tau_{2}=2, g_{1}=1$, $g_{2}=2, \eta=10, \rho=5$, and other necessary parameters or functions are the same as that of the above simulation. The corresponding adaptive law, sliding mode, and controller are chosen as follows according to [12]:

$$
\begin{aligned}
& \dot{\tilde{\theta}}_{1}=-\tau_{1}\left(p_{2} x_{1}+p_{3} x_{2}\right) \sin \left(x_{1}\right), \\
& \dot{\tilde{\theta}}_{2}=-\tau_{2}\left(p_{2} x_{1}+p_{3} x_{2}\right) \cos \left(x_{1}\right) \text {, } \\
& x_{3_{d}}=-g_{1} x_{1}-g_{2} x_{2}+\widetilde{\theta}_{1} \sin \left(x_{1}\right)+\widetilde{\theta}_{2} \cos \left(x_{1}\right) \text {, } \\
& \sigma=x_{3}-x_{3_{d}} \text {, } \\
& u=-\left[\eta \sigma+p_{2} x_{1}+p_{3} x_{2}-x_{3}-\left(-g_{1}+\cos \left(x_{1}\right) \widetilde{\theta}_{1}\right) \dot{x}_{1}\right. \\
& -\left(-g_{2}-\sin \left(x_{1}\right) \widetilde{\theta}_{2}\right)\left(x_{3}-\widetilde{\theta}_{1} \sin \left(x_{1}\right)-\widetilde{\theta}_{2} \cos \left(x_{1}\right)\right) \\
& \left.-\sin \left(x_{1}\right) \dot{\tilde{\theta}}_{1}-\cos \left(x_{1}\right) \dot{\tilde{\theta}}_{2}\right]-\rho \operatorname{sgn}(\sigma) \text {. }
\end{aligned}
$$
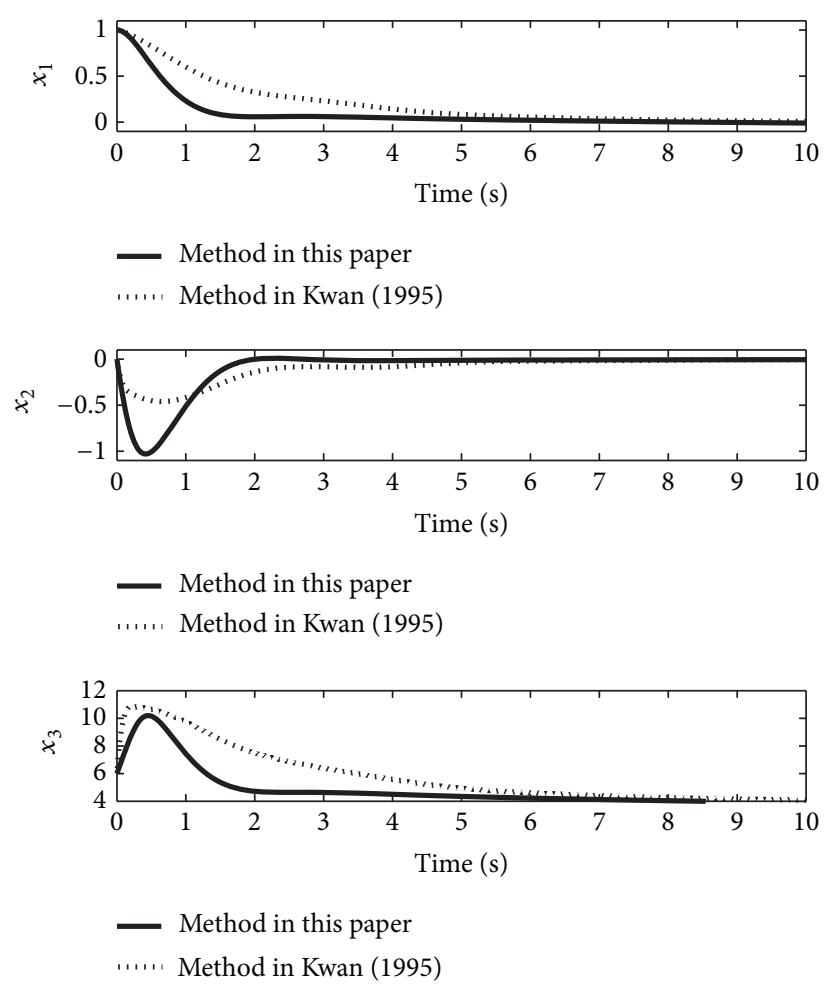

FIgURE 1: Comparison about states $x_{1}, x_{2}$, and $x_{3}$.

From the comparison, one can find that, under the presented UDE-based ASMC, the states converge faster than that of [12] method. The amplitude of input is especially around 20 at the beginning and the input is without chattering in our method; however, the maximal amplitude of input is greater than 80 and the input is with strong chattering in [12] method. Although state $x_{2}$ is a bit larger in our method, [12] method requires about 10 seconds to force state $x_{2}$ to arrive origin; on the contrary, state $x_{2}$ can converge in 3 seconds under the presented method. Therefore, the created UDE-based ASMC method in this paper is better on the whole (see Table 1).

\section{Conclusions}

In this paper, a novel uncertainty and disturbance estimatorbased adaptive sliding mode control (UDE-based ASMC) method is presented for nonlinear systems, which are with parameter uncertainties as well as unmatched model uncertainties and external disturbances. Parameter estimation law is obtained according to immersion and invariance (I\&I) adaptive control approach, and uncertainty and disturbance estimator is employed to realize chattering elimination in essence. UDE-based ASMC can guarantee closed-loop systems that have strong robustness without requiring to know the bounds of uncertainties. At the end of this paper, UDEbased ASMC is applied to a pendulum with motor dynamics, simulation results illustrate the approving performance, including chattering elimination, control peak decreasing, fast convergence, and strong robustness to parameter uncertainties and external uncertainties. 
TABLE 1: Comparison between the two methods.

\begin{tabular}{|c|c|c|c|c|c|}
\hline & & $x_{1}$ & $x_{2}$ & $x_{3}$ & $u$ \\
\hline \multirow{2}{*}{ Method in this paper } & Peak value & 1 & -1.0301 & 10.2015 & 20.9345 \\
\hline & Converge time & 2 & 3 & 2.5 & 3 \\
\hline \multirow[t]{2}{*}{ Method in Kwan (1995) [12] } & Peak value & 1 & -0.4604 & 10.8797 & 81.243 \\
\hline & Converge time & 7 & 6 & 8 & $\infty$ \\
\hline
\end{tabular}

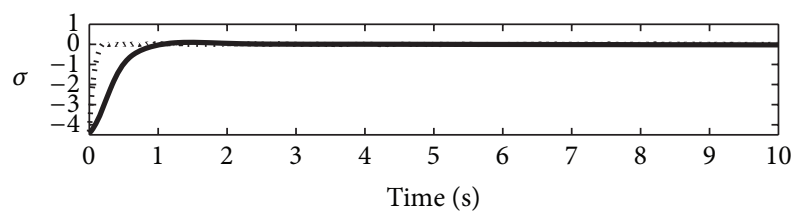

- Method in this paper ....... Method in Kwan (1995)
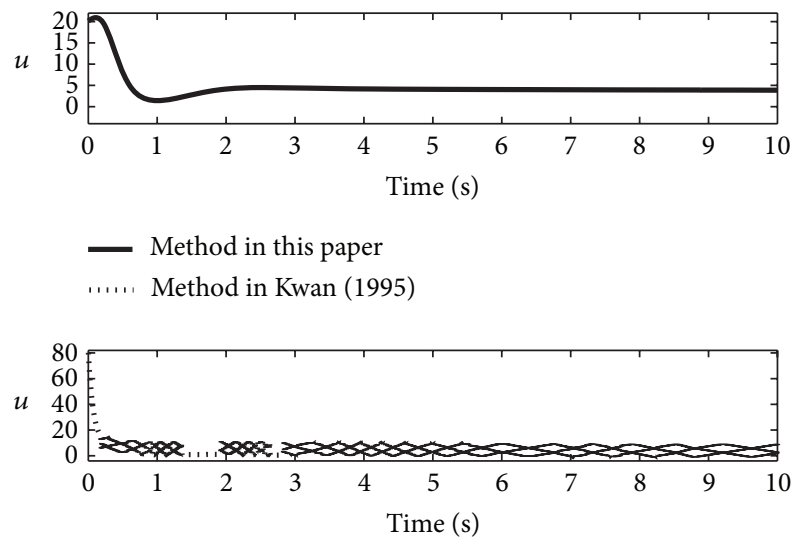

- Method in this paper

....... Method in Kwan (1995)

FIGURE 2: Comparison about sliding mode $\sigma$ and control signal $u$.

\section{Conflict of Interests}

The authors declare that there is no conflict of interests regarding the publication of this paper.

\section{Acknowledgments}

This work was supported by the Natural Science Foundation of China (51275249) and the Talent Introduction Foundation of Engineering College Nanjing Agricultural University (Rcqd11-06).

\section{References}

[1] V. I. Utkin, Sliding Modes in Control and Optimization, Springer, Berlin, Germany, 1992.

[2] G. Bartolini, A. Ferrara, E. Usai, and V. I. Utkin, "On multiinput chattering-free second-order sliding mode control," IEEE Transactions on Automatic Control, vol. 45, no. 9, pp. 1711-1717, 2000.
[3] I. Boiko, L. Fridman, A. Pisano, and E. Usai, "Analysis of chattering in systems with second-order sliding modes," IEEE Transactions on Automatic Control, vol. 52, no. 11, pp. 2085-2102, 2007.

[4] M. Taleb, A. Levant, and F. Plestan, "Pneumatic actuator control: Solution based on adaptive twisting and experimentation," Control Engineering Practice, vol. 21, no. 5, pp. 727-736, 2013.

[5] Y. Shtessel, M. Taleb, and F. Plestan, "A novel adaptive-gain supertwisting sliding mode controller: methodology and application," Automatica, vol. 48, no. 5, pp. 759-769, 2012.

[6] S. Kurode, S. K. Spurgeon, B. Bandyopadhyay, and P. S. Gandhi, "Sliding mode control for slosh-free motion using a nonlinear sliding surface," IEEE/ASME Transactions on Mechatronics, vol. 18, no. 2, pp. 714-724, 2013.

[7] V. I. Utkin and A. S. Poznyak, "Adaptive sliding mode control with application to super-twist algorithm: equivalent control method," Automatica, vol. 49, no. 1, pp. 39-47, 2013.

[8] A. Levant, "Sliding order and sliding accuracy in sliding mode control," International Journal of Control, vol. 58, no. 6, pp. 12471263, 1993.

[9] G. Bartolini, A. Ferrara, and V. I. Utkin, "Adaptive sliding mode control in discrete-time systems," Automatica, vol. 31, no. 5, pp. 769-773, 1995.

[10] H. Lee and V. I. Utkin, "Chattering suppression methods in sliding mode control systems," Annual Reviews in Control, vol. 31, no. 2, pp. 179-188, 2007.

[11] F. Plestan, Y. Shtessel, V. Brégeault, and A. Poznyak, "New methodologies for adaptive sliding mode control," International Journal of Control, vol. 83, no. 9, pp. 1907-1919, 2010.

[12] C.-M. Kwan, "Sliding mode control of linear systems with mismatched uncertainties," Automatica, vol. 31, no. 2, pp. 303-307, 1995.

[13] B. Yao and M. Tomizuka, "Adaptive robust control of MIMO nonlinear systems in semi-strict feedback forms," Automatica, vol. 37, no. 9, pp. 1305-1321, 2001.

[14] A. C. Huang and Y. H. Chen, "Adaptive multiple-surface sliding control for non-autonomous systems with mismatched uncertainties," Automatica, vol. 40, no. 11, pp. 1939-1945, 2004.

[15] L. Xiao, H. Su, and J. Chu, "Sliding mode prediction based control algorithm for discrete-time non-linear uncertain coupled systems," International Journal of Control, vol. 80, no. 10, pp. 1616-1625, 2007.

[16] Q. Hu, G. Ma, and L. Xie, "Robust and adaptive variable structure output feedback control of uncertain systems with input nonlinearity," Automatica, vol. 44, no. 2, pp. 552-559, 2008.

[17] X. Chen and T. Hisayama, "Adaptive sliding-mode position control for piezo-actuated stage," IEEE Transactions on Industrial Electronics, vol. 55, no. 11, pp. 3927-3934, 2008.

[18] X. Han, E. Fridman, and S. K. Spurgeon, "Sliding-mode control of uncertain systems in the presence of unmatched disturbances with applications," International Journal of Control, vol. 83, no. 12, pp. 2413-2426, 2010. 
[19] Z. Liu, H. Su, and S. Pan, "A new adaptive sliding mode control of uncertain nonlinear systems," Asian Journal of Control, vol. 16, no. 1, pp. 198-208, 2014.

[20] Q.-C. Zhong and D. Rees, "Control of uncertain LTI systems based on an uncertainty and disturbance estimator," Journal of Dynamic Systems, Measurement and Control-Transactions on ASME, vol. 126, no. 4, pp. 905-910, 2004.

[21] S. E. Talole and S. B. Phadke, "Model following sliding mode control based on uncertainty and disturbance estimator," Journal of Dynamic Systems, Measurement and Control, vol. 130, no. 3, Article ID 034501, 5 pages, 2008.

[22] H. K. Khalil, Nonlinear Systems, Prentice Hall, 2002.

[23] A. Astolfi, D. Karagiannis, and R. Ortega, Nonlinear and Adaptive Control with Applications, Springer, London, UK, 2008. 


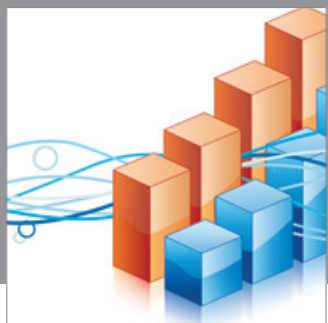

Advances in

Operations Research

mansans

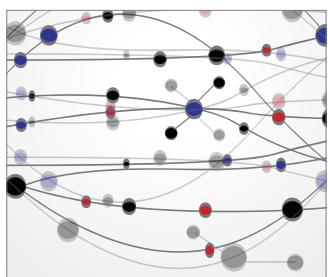

The Scientific World Journal
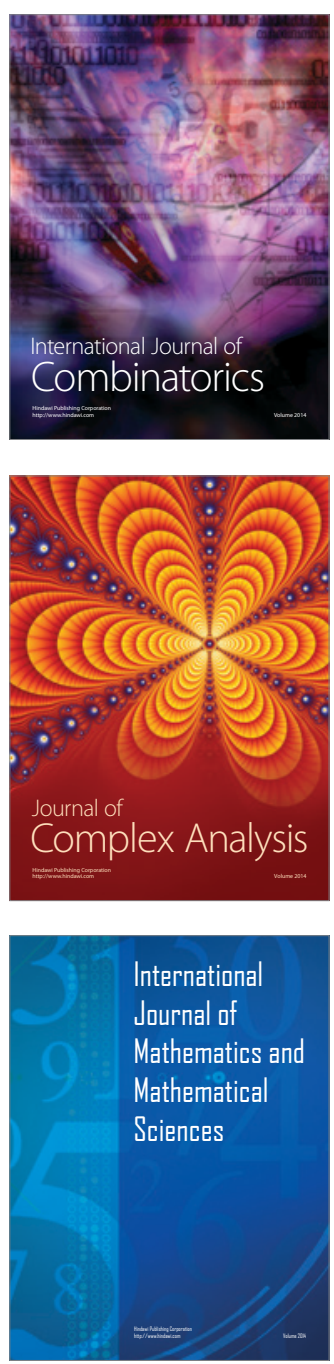
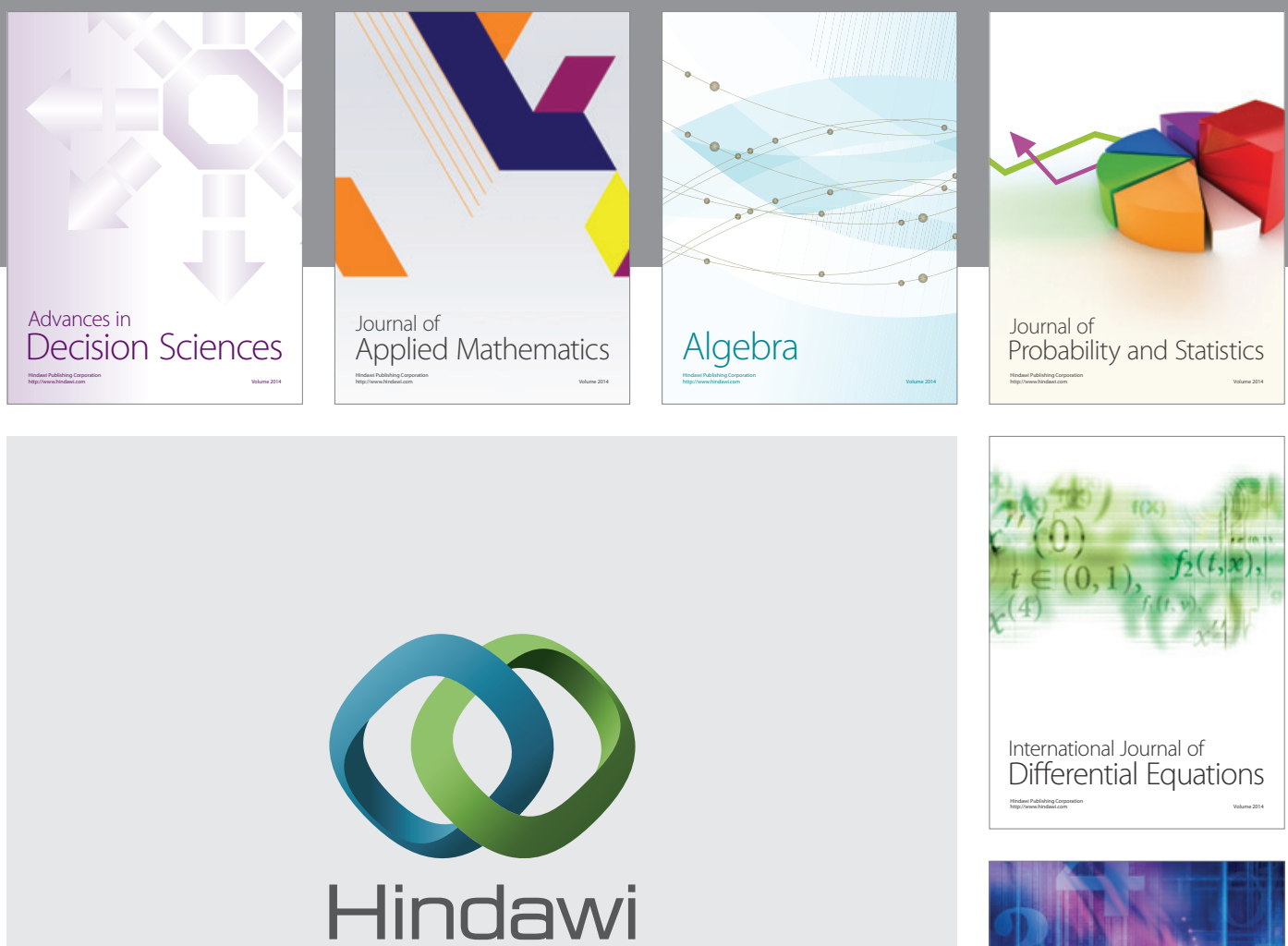

Submit your manuscripts at http://www.hindawi.com
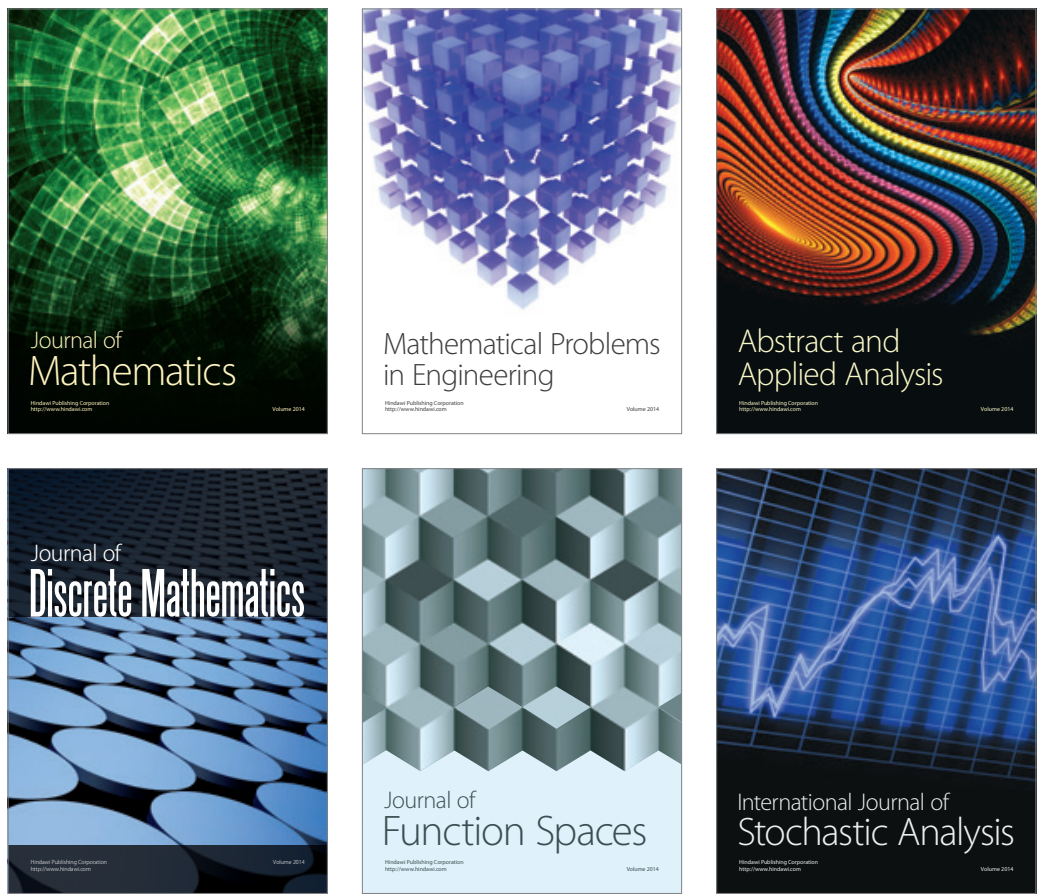

Journal of

Function Spaces

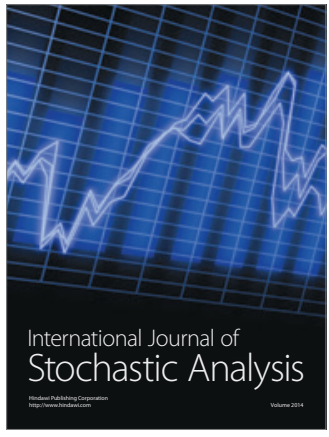

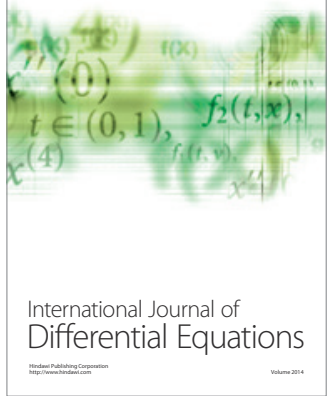
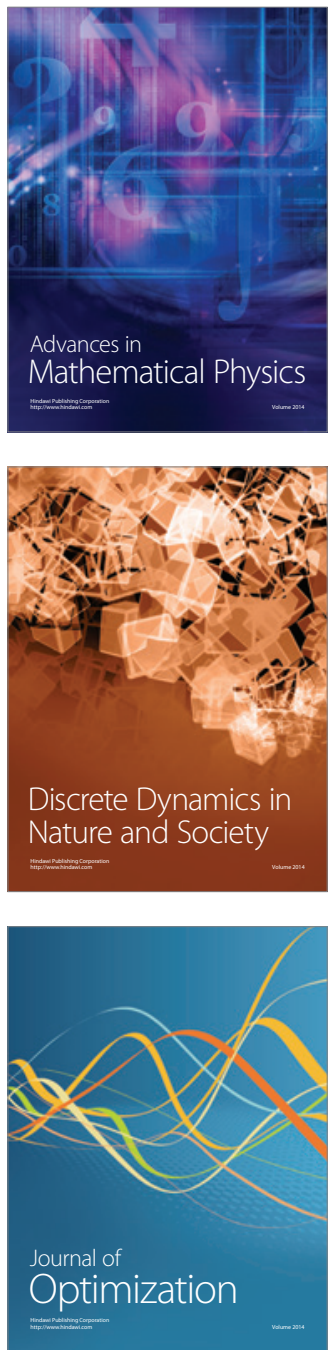